\section{Eosinophile Bronchitis} ohne Asthma - Eine weitere, seltene Ursache des chronisch persistierenden Hustens (CPH)?

\author{
Ein 30-jähriger Patient mit schwerem $\mathrm{CPH}$ \\ infolge einer eosinophilen Bronchitis \\ ohne Asthma oder Hyperreagibilität
}

Zusammenfassung: CPH (Husten ohne evidente radiologische oder lungenfunktionsanalytisch fassbare Ursache) kann oft an eine bronchiale Hyperreagibilität (Husten als Asthmaäquivalent), banale Erkrankungen der oberen Atemwege (Rhinopathie, Sinusitis) gastroösophagealen Reflux oder an ACE-(angiotensin converting enzyme)Hemmer Einnahme zurückgeführt und entsprechend therapiert werden. Trotz sorgfältiger Diagnostik bleibt aber die Ursache des $\mathrm{CPH}$ in $10 \%$ der Fälle unklar. Es wird über einen 30-jährigen Patienten mit sehr starkem $\mathrm{CPH}$ berichtet (bei Erwachsenen ungewöhnlich: er musste sich wiederholt übergeben). Die Thoraxaufnahme war nicht diagnostisch. Für eine Rhinopathie bestand kein Anhalt. Er war weder obstruktiv noch hyperreagibel im Metacholintest, die 24-Stunden-pH-Metrie war negativ. Für Keuchhusten fand sich serologisch kein Anhalt. Die Bronchoskopie blieb ebenfalls unauffällig. Die Schleimhautbiopsie ergab jedoch zahlreiche eosinophile Granulozyten in der Schleimhaut und eine deutliche Verdickung der Basalmembran. Im Sputum fand sich eine Eosinophilie von $21 \%$. Der Patient war nach einwöchiger inhalativer Kortisontherapie mit Budesonid beschwerdefrei geworden. Die Therapie konnte nach 3 Wochen abgesetzt werden, es kam nicht zum Rezidiv. In der Literatur wurde in den letzten Jahren die eosinophile Bronchitis (ohne Asthma und ohne Hyperreagibilität) mit gutem Ansprechen auf inhalative Steroide als mögliche Ursache des CPH bei wenigen Patienten beschrieben. Husten als Asthmaäquivalent („cough type asthma“) und die eosinophile Bronchitis könnten rudimentäre (forme fruste) klinische Präsentationen des Asthma bronchiale - jeweils ohne klassisches Pfeifen und Brummen sowie ohne Atemnot - darstellen. In beiden Fällen handelt es sich um ein $\mathrm{CPH}$, der wie Asthma prompt auf inhalative Kortikosteroide reagiert. Daneben weisen Patienten mit Husten als Asthmaäquivalent aus der Bandbreite des Asthmaphänotyps die asthmatypische Hyperreagibilität, Patienten mit eosinophiler Bronchitis hingegen Sputum- und Schleimhauteosinophilie sowie die Verdickung der Basalmembran auf.

Eosinophilic Bronchitis Without Asthma - An Additional Rare Cause For Chronic Persistent Cough (CPC)? 30 Year Old Patient With Severe CPC Due To Eosinophilic Bronchitis: In non-smokers the underlying causes for chronic persistent cough (CPC) e.g. chronic cough without diagnostic chest X-ray or pulmonary function test - are usually as follows: several common upper airways diseases, bronchial (cough type) asthma,

Pneumologie 2001; 55: 249-252

(c) Georg Thieme Verlag Stuttgart · New York

ISSN 0934-8387

\section{P. Kardos}

Gemeinschaftspraxis und Pneumologisches-allergologisches

Zentrum, Maingau Krankenhaus, Frankfurt a.M. gastrooesophageal reflux or treatment with an ACE (angiotensin converting enzyme) - inhibitor. In $10 \%$ of CPC however the cause remains uncertain. We report a 30 year old non-smoker with severe coughing and repeated vomiting for two months. No laboratory or technical data could be collected suggestive of a common cause of CPC: Upper airways disease, bronchial flow limitation or hyperresponsiveness, ACE inhibitor medication, B. pertussis infection, gastrooesophageal reflux disease (by 24hours pH-probe) were ruled out. Fiberbronchoscopic findings remained unremarkable, except for the bronchial biopsy specimen, which showed moderate eosinophilic inflammation of the mucosa and marked thickening of the subepithelial layer. Since the cough was non-productive, sputum induction with $3 \mathrm{ml}$ nebulised $3 \% \mathrm{NaCl}$ solution was performed. $28 \%$ of the granulocytes were eosinophil stained. A low quality morning sputum $(<1 \mathrm{ml})$ showed $21 \%$ eosinophilia. Thus, the diagnosis of eosinophilic bronchitis was established. $400 \mu \mathrm{g}$ budesonide dry powder inhalations b.i.d. for one week resolved the cough, treatment was stopped after three weeks. No recurrence was seen two months later. Both the cough type asthma and the eosinophilic bronchitis could represent a forme fruste of classical bronchial asthma beyond wheezing or dyspnoea, but with the common main symptom: cough. Since hyperresponsiveness and cough are phenotypic hallmarks of cough variant asthma, in eosinophilic bronchitis - beside cough - another two features of asthma are present: eosinophilic inflammation of the mucosa along with sputum eosinophilia and subepithelial layer thickening. Not surprisingly, eosinophilic bronchial inflammation could be shown in patients with cough variant asthma as well, who - up to $56 \%$ during a four year-period - develop classic asthma. The long-term outcome of eosinophilic bronchitis is not known, however. Thus, asthma, cough variant asthma and cough due to eosinophilic bronchitis can mirror different phenotypes or phases of the same entity. CPC due to either the cough type asthma or the eosinophilic bronchitis is like asthma fast responding to inhalative steroids. (Induced) sputum staining should be added to the diagnostic armamentarium of CPC.

\section{Einleitung}

Husten ist sehr oft Auslöser von Arzt-Patient-Kontakten. Am häufigsten handelt es sich um einen akuten Husten, die meist zugrunde liegende akute virale Bronchitis heilt spätestens nach drei bis vier Wochen spontan aus. Ein subakuter, bis zu acht (bis zwölf) Wochen anhaltender Husten kann nach bestimmten bronchopulmonalen Infekten (Pertussis, Mykoplasmen- oder Adenovirusinfekte) infolge Schädigung des 
Bronchialepithels, postviraler Bronchiolitis oder einer vorübergehenden Erhöhung der bronchialen Reaktionsbereitschaft entstehen. Der chronische über acht Wochen anhaltende Husten ist oft Symptom definierter Lungenerkrankungen [Asthma, COPD (chronic obstructive pulmonary disease), interstitielle Lungenerkrankungen, Tuberkulose, Bronchialkarzinom]. Die Röntgen-Thoraxaufnahme und die Lungenfunktionsprüfung weisen meist die Richtung zur Diagnose. Manche Patienten aber husten chronisch ohne radiologisch und lungenfunktionsanalytisch evidente Ursache (chronisch persistierender Husten CPH) [1], obwohl sie Nichtraucher sind. Ihr Husten kann oft erfolgreich nach dem anatomisch-diagnostischen Protokoll [2] abgeklärt werden. Die häufigsten Ursachen für einen solchen chronischen Husten sind: banale entzündliche Erkrankungen der oberen Atemwege, Husten als Asthmaäquivalent sowie gastroösophagealer Reflux oder die Einnahme von ACE-(angiotensin converting enzyme)Hemmer [3]. Bei mindestens $10 \%$ der $\mathrm{CPH}$ Patienten lässt sich die Ursache des Hustens trotz exzessiver Diagnostik nicht abklären [4-6]. Die eosinophile Bronchitis als seltene Ursache des $\mathrm{CPH}$ wurde erst in den letzten Jahren von zwei Arbeitsgruppen bei wenigen Patienten beschrieben [7-9]. Wir stellen einen solchen Patienten vor.

\section{Kasuistik}

Der 30-jährige Patient klagte über seit 2 Monaten anhaltenden starken, oft nächtlichen Husten. Er huste so stark, dass er sich manchmal übergeben müsse. Er habe vor drei Jahren bereits einen wochenlang anhaltenden Husten nach einem Virusinfekt gehabt. Als Kind habe er an Heuschnupfen gelitten, sei desensibilisiert worden und habe seit zehn Jahren keinen Heuschnupfen mehr gehabt. Eine Vorbehandlung mit Roxigrün ${ }^{\circledR}$ und Codipront ${ }^{\circledR}$ habe nicht geholfen. Der Patient ist Nichtraucher.

Bei der körperlichen Untersuchung fanden sich keine Auffälligkeiten. Die Nasenatmung war völlig frei, die Nasenschleimhaut unauffällig, keine Pharyngitis. Auskultationsbefund über den Lungen war ebenfalls unauffällig.

Die Thoraxaufnahme fiel normal aus und erklärte den Husten nicht.

Die Lungenfunktionsprüfung ergab eine Vitalkapazität von $94 \%$, die Einsekundenkapazität $\left(\mathrm{FEV}_{1}\right)$ betrug $100 \%$ des Sollwertes. Somit wurde eine manifeste Bronchialobstruktion ausgeschlossen. Es wurde eine unspezifische inhalative Provokationstestung mit der Dosimetermethode (APS Jaeger, Würzburg) durchgeführt. Die Metacholinschwelle für einen 100\%-igen Anstieg der ganzkörperplethysmographisch gemessenen spezifischen Resistance lag höher, als 5,25 mg vernebelte Dosis von Metacholin (2,5\%ige Lösung). Eine bronchiale Hyperreagibilität, wie man sie beim Husten als Asthmaäquivalent sieht, wurde damit ausgeschlossen.

Laborbefunde: Blutsenkung $2 \mathrm{~mm} \mathrm{n}$. W., C-reaktives Protein $<5 \mathrm{mg} / \mathrm{l}$, Leukozytenzahl: $6100 / \mathrm{mm}^{3}$. Differenzialausstrich: $70 \%$ Neutrophile, $22 \%$ Lymphozyten, 7\% Monozyten, $1 \%$ Eosinophile.
Es handelte sich um einen $\mathrm{CPH}$, dessen Ursache trotz einer sorgfältig erhobenen Anamnese, körperlicher Untersuchung und Durchführung der Routinediagnostik ungeklärt blieb.

Angesichts der Heuschnupfenanamnese dachten wir an Husten als Asthmaäquivalent, die negative bronchiale Provokationstestung schloss aber diese Diagnose aus. Eine relativ häufige Ursache des chronisch persistierenden Hustens ist der gastroösophageale Reflux. Ältere Patienten haben dabei oft kein Sodbrennen, bei Jüngeren ist das Sodbrennen eher richtungweisend. Unser Patient hatte auch auf Befragen kein häufiges (öfter, als einmal pro Woche auftretendes ) Sodbrennen angegeben. Die 24-Stunden-pH-Metrie ergab eine Säureexpositionszeit von 2,2\% der Gesamtzeit (normal bis 3,4\%). Eine Assoziation zwischen Husten und Refluxepisoden fand sich nicht.

Die Pricktestung im Juni mit den 15 häufigsten inhalativen Allergenen ergab schwach positive Reaktionen (jeweils $3 \mathrm{~mm}$ bei einer Histaminquaddel von $5 \mathrm{~mm}$ ) auf Birken- und Gramineenpollen.

Die Bronchoskopie zeigte unauffällige Ostien, auch bei Husten oder forcierter Atmung keine Zeichen einer Bronchialwandinstabilität. Die Schleimhaut war unauffällig. In der Bronchiallavage fanden sich nur vereinzelt Keime ( $H$. influenzae, Streptococcus, nicht gruppierbar und Neisseria species).

Die histologische Untersuchung der Schleimhautbioptate aus dem Mittellappen (Abb.1) zeigte eine deutlich verbreiterte Basalmembran und reichlich eosinophile Infiltrate der Schleimhaut. Der Patient konnte zunächst kein Sputum produzieren. Er hatte einen definitionsgemäß trockenen Husten (deutlich weniger, als $30 \mathrm{ml}$ Auswurf/24 Stunden), so dass nach Vorliegen der Ergebnisse der Histologie zunächst das induzierte Sputum (Inhalation von $3 \mathrm{ml} 3 \%$ ige Kochsalzlösung mit dem Pari Turboboy ${ }^{\mathrm{TM}}$ ) untersucht wurde. Wir fanden $28 \%$ Eosinophile. Die qualitative mikroskopische Untersuchung des zwei Tage später (noch ohne Therapie) nachgereichten, für eine Aussage normalerweise nicht geeigneten Morgen-

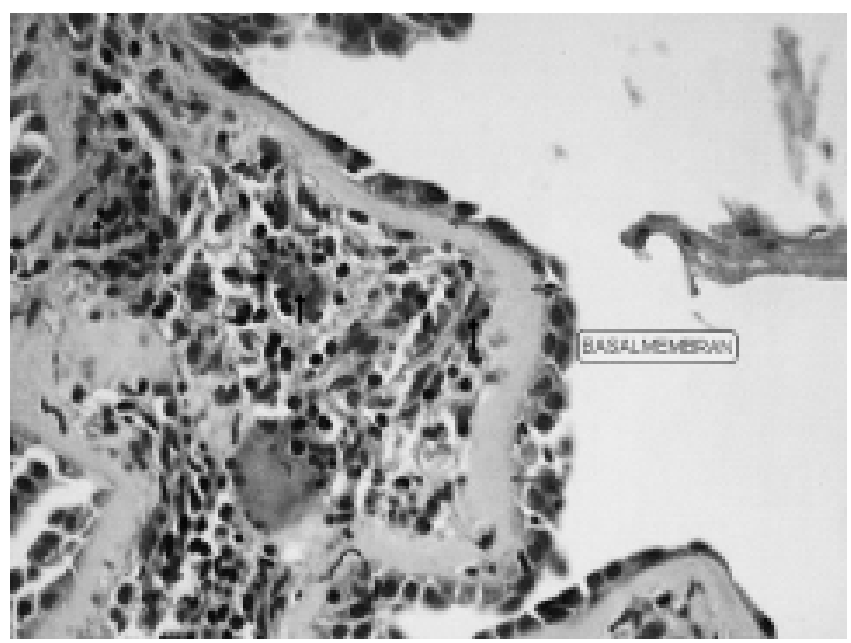

Abb. 1 Bronchialschleimhautbiopsie bei eosinophiler Bronchitis (Hematoxylin-Eosin-Färbung). Die Pfeile zeigen eosinophile Granulozyten. Die Basalmembran ist auf 7-10 $\mu$ verdickt. 
sputums: ( $<1 \mathrm{ml}, 10-15$ Epithelzellen, $<25$ Leukozyten pro Sichtfeld): Eosinophilie von $21 \%$.

Damit wurde die Diagnose einer eosinophilen Bronchitis gestellt.

Die Therapie erfolgte mit $2 \times 400 \mu$ gudesonid aus dem Pulverinhalator. Der Patient wurde nach einer Woche Therapie völlig beschwerdefrei. Die Behandlung wurde über drei Wochen fortgesetzt. Er blieb zwei Monate nach Absetzen des inhalativen Steroids symptomfrei.

\section{Diskussion}

Der Patient klagte - 10 Jahre nachdem er das letzte Mal Heuschnupfen gehabt hat - während der Pollenflugzeit über sehr starken Husten. Heuschnupfen, Konjunktivitis oder Asthma traten nicht auf. Eine exogen allergische Genese der histologisch und sputumzytologisch gefundenen Eosinophilie erscheint wenig wahrscheinlich, da ein (allergisches) Asthma bei dem nicht therapierten Patienten weder klinisch (fehlende Atemnot, fehlendes Pfeifen und Brummen) noch durch Lungenfunktionsprüfung (fehlende Obstruktion, fehlende Hyperreagibilität) wahrscheinlich gemacht werden konnte.

Gibson et al. [8] beschrieben bereits 1989 in Lancet ein kleines Kollektiv von sieben Patienten, die ähnlich, wie Asthmatiker im Sputum vermehrt Eosinophile hatten. Sie litten nicht an Asthma und hatten im Metacholintest - wie unser Patient keine bronchiale Hyperreagibilität. Alle sieben sprachen auf eine Kortikosteroidtherapie an. In einer weiteren Gruppe von 9 Patienten mit Husten und Eosinophile fand die gleiche Gruppe [9] nur bei drei Patienten eine plateauförmig begrenzte Metacholin-Hyperreagibilität und nur bei einem asthmatypische Peak-flow-Variationen. Sie folgern, dass Sputum-Eosinophilie nicht notwendigerweise nur bei Asthma auftreten kann. Brightling et al. [7] fanden 1999 unter 856 wegen Husten überwiesenen Patienten 18 Fälle mit eosinophiler Bronchitis. Auch diese Patienten hatten eine normale bronchiale Reaktionsbereitschaft und keine Asthmasymptome gehabt. Sie sprachen klinisch auf inhalatives Budesonid sehr gut an. Auch die Anzahl der Eosinophilen im Sputum ging unter dieser Therapie zurück.

Der Ausdruck eosinophile Bronchitis wurde in der Vergangenheit gelegentlich als pathogenetisch orientierte Beschreibung des Asthma bronchiale (in Abgrenzung zu COPD: neutrophile Bronchitis) benützt. Beim Asthma handelt es sich definitionsgemäß neben variabler Bronchialobstruktion und bronchialer Hyperreagibilität um eine durch Dominanz der eosinophilen Granulozyten charakterisierte Entzündung der Bronchialschleimhaut. Typisch ist bei chronischem Asthma auch die Verdickung der Basalmembran („remodelling“). Husten ist eines der Kardinalsymptome des Asthmas. Es gibt ein rudimentäres Asthma, charakterisiert nur durch Husten und Hyperreagibilität: Husten als Asthmaäquivalent, in der englischsprachigen Literatur „cough type asthma“ oder „,ough variant asthma“ genannt. [10]. Bei diesem rudimentären Asthma wurde allerdings auch eine eosinophile Entzündung [12] neuerdings sogar „remodelling“ der Schleimhaut [13] beschrieben. Bei manchen Patienten mit Husten als Asthmaäquivalent treten sogar asthmatypische Peak-flow-Schwankungen auf [14].
Es wurde in den letzten Jahren neben Husten als Asthmaäquivalent eine weitere Form des $\mathrm{CPH}$ mit eosinophiler Bronchitis beschrieben $[7,8]$, wie bei unserem Patienten. Hierbei fehlen zum klassischen Asthmaphänotyp nicht nur die Atemnot, das Pfeifen und Brummen sondern auch noch die Hyperreagibilität. Die eosinophile Entzündung und bei unserem Patienten überraschenderweise auch die Verdickung der Basalmembran (remodelling) sind jedoch nachweisbar: die Basalmembrandicke betrug $7-10 \mu$ gegenüber dem Normalbefund von 3-5 $\mu$ [20]. Bei Asthmatikern wie bei solchen CPH-Patienten fanden sich in der bronchoalveolären Lavage identische messenger Ribonucleinsäuren (mRNA's) für die Expression von Interleukin-5 und GM-CSF (granulocyte-macrophage colony stimulating factor) [15], somit dürfte der Pathomechanismus der eosinophilen Entzündung bei beiden Krankheitsbildern der gleiche sein. Inwieweit dieses Krankheitsbild eventuell später zum Husten als Asthmaäquivalent führt (mit anderen Worten: auf dem Boden der eosinophilen Entzündung Hyperreagibilität hinzutritt) oder gar in das klassische Asthma mündet, ist zur Zeit noch nicht klar. Der Übergang von Husten als Asthmaäquivalent zum klassischen Asthma ist hingegen fließend: $17-56 \%$ der Fälle bekommen später ein klassisches Asthma $[15,16]$. Im Gegensatz zur in der Literatur vorherrschenden Ansicht, dass CPH infolge eosinophiler Bronchitis und Husten als Asthmaäquivalent getrennte Entitäten seien [17], könnten die eosinophile Bronchitis und Husten als Asthmaäquivalent eher zwei Formen des Asthmaphänotyps oder zwei Stadien der gleichen Entität: des klassischen Asthma bronchiale darstellen.

Wie Husten als Asthmaäquivalent spricht auch die eosinophile Bronchitis sehr gut auf inhalatives Kortison an, bereits eine einwöchige Behandlung mit höchstens mittelhoch dosiertem inhalativen Kortison $(800 \mu \mathrm{g}$ Beclomethasondipropionat) [9] bzw. Budesonid [7] reicht wie bei unserem Patienten aus, um sowohl den Husten als auch die Eosinophilie zum Abklingen zu bringen.

Ein weiterer höchst bemerkenswerter Aspekt ist die Basalmembranverdickung (beginnendes remodelling) bei unserem Patienten, der niemals asthmatische Symptome hatte, obstrukionsfrei, ja sogar nicht einmal hyperreagibel war. „Remodelling" wurde auch bei Husten als Asthmaäquivalent beschrieben [13]. Die Verdickung der Basalmembran kann in diesem Fall nur als ein zytokinvermittelter eosinophiler Entzündungsprozess angesehen werden, welcher bereits vor dem asthmatypischen Epithelschaden auftritt und mit obstruktiver Atemmechanik offensichtlich nichts zu tun hat. In dieser Hinsicht bleibt zu diskutieren, ob die inhalative Kortisontherapie nicht doch viel länger, als sie zur reinen Symptomkontrolle erforderlich war, verordnet werden sollte.

Die Untersuchung des ggf. induzierten Sputums auf Eosinophile muss in das diagnostische Arsenal [19] des CPH aufgenommen werden. Eine Bronchoskopie scheint bei negativem Röntgen-Thoraxbefund, Sputumeosinophilie und schnellem Ansprechen auf eine inhalative Kortisontherapie nicht erforderlich zu sein. 


\section{Literatur}

${ }^{1}$ Kardos P. Chronisch Persistierender Husten (CPH). Pneumologie 1995; 49: $2-13$

2 Irwin RS, Rosen MJ, Braman SS. Cough. A Comprehensive Review. Arch Intern Med 1977; 137: 1186-1191

${ }^{3}$ Irwin RS, Curley FJ, French CL. Chronic Cough. The Spectrum and Frequency of Causes, Key Components of the Diagnostic Evaluation, and Outcome of Specific Therapy. Am Rev Respir Dis 1990; 141: $640-647$

${ }^{4}$ Kardos P, Gebhardt T. Chronisch persistierender Husten (CPH) in der Praxis: Diagnostik und Therapie bei 329 Patienten in 2 Jahren. Pneumologie 1996; 50: 437-441

${ }^{5}$ Palombini BC, Villanova CA, Araújo E, Gastal OL, Alt DC, Stolz DP, Palombini CO. A Pathogenic Triad in Chronic Cough: Asthma, Postnasal Drip Syndrome, and Gastroesophageal Reflux Disease. Chest. 1999; 116: 279-284

${ }^{6}$ McGarvey LP, Heaney LG, Lawson JT, Johnston BT, Scally CM, Ennis M. Evaluation and Outcome of Patients With Chronic NonProductive Cough Using a Comprehensive Diagnostic Protocol. Thorax 1998; 53: 738 - 743

${ }^{7}$ Brightling CE, Ward R, Goh KL, Wardlaw AJ, Pavord ID. Eosinophilic Bronchitis Is an Important Cause of Chronic Cough. Am J Respir Crit Care Med 1999; 160: 406-410

${ }^{8}$ Gibson PG, Dolovich J, Denburg J, Ramsdale EH, Hargreave FE. Chronic Cough: Eosinophilic Bronchitis Without Asthma. Lancet 1989; $1: 1346-1348$

${ }^{9}$ Gibson PG, Hargreave FE, Girgis-Gabardo A, Morris M, Denburg JA, Dolovich J. Chronic Cough With Eosinophilic Bronchitis: Examination for Variable Airflow Obstruction and Response to Corticosteroid. Clin Exp Allergy 1995; 25: 127-132

${ }^{10}$ Johnson D, Osborn LM. Cough Variant Asthma: A Review Of the Clinical Literature. J Asthma 1991; 28: 85-90

${ }^{11}$ Koh YY, Chae SA, Min KU. Cough Variant Asthma Is Associated With a Higher Wheezing Threshold Than Classic Asthma. Clin Exp Allergy 1993; 23: 696-701

12 Niimi A, Amitani R, Suzuki K, Tanaka E, Murayama T, Kuze F. Eosinophilic Inflammation in Cough Variant Asthma. Eur Respir J 1998; 11: $1064-1069$

${ }^{13}$ Niimi A, Matsumoto H, Minakuchi M, Kitaichi M, Amitani R. Airway Remodelling in Cough-Variant Asthma [Letter]. Lancet 2000; 356: 564-565

14 Tokuyama K, Shigeta M, Maeda S, Takei K, Hoshino M, Morikawa A. Diurnal Variation of Peak Expiratory Flow in Children With Cough Variant Asthma. J Asthma 1998; 35: 225-229

${ }^{15}$ Gibson PG, Zlatic K, Scott J, Sewell W. Chronic Cough Resembles Asthma With IL-5 and Granulocyte-Macrophage Colony-Stimulating Factor Gene Expression in Bronchoalveolar Cells. J Allergy Clin Immunol 1998; 101: 320

${ }^{16}$ Iwanaga T, Inuzuka S, Takahashi N, Kishikawa R, Yokota K, Ikeda T, Hirose T, Tsurutani H, Nishima S. Cough Variant Asthma Among Patients With Chronic Persistent Cough and Its Clinical Outcome. Arerugi 1998; 47: 457-461 [Abstract]

${ }^{17}$ Koh YY, Jeong JH, Park Y, Kim CK. Development of Wheezing in Patients With Cough Variant Asthma During an Increase in Airway Responsiveness. Eur Respir J 1999; 14: 302 - 308

${ }^{18}$ Irwin RS, Madison JM. Review Articles: Primary Care: The Diagnosis and Treatment of Cough. N Engl J Med 2000; 343: $1715-1721$

${ }^{19}$ Kardos P. Vorschläge für eine rationale und rationelle Diagnostik des Hustens. Pneumologie 2000; 54: 110- 115

${ }^{20}$ Wilson JW, Li X. The measurement of reticular basement membrane and submucosal collagen in the asthmatic airway. Clin Exp Allergy 1997; 27: 363 - 371
Dr. med. P. Kardos

Gemeinschaftspraxis und Pneumologisches-allergologisches Zentrum Maingau Krankenhaus

Scheffelstraße 33

60318 Frankfurt am Main

E-mail: peter.kardos@netsurf.de 